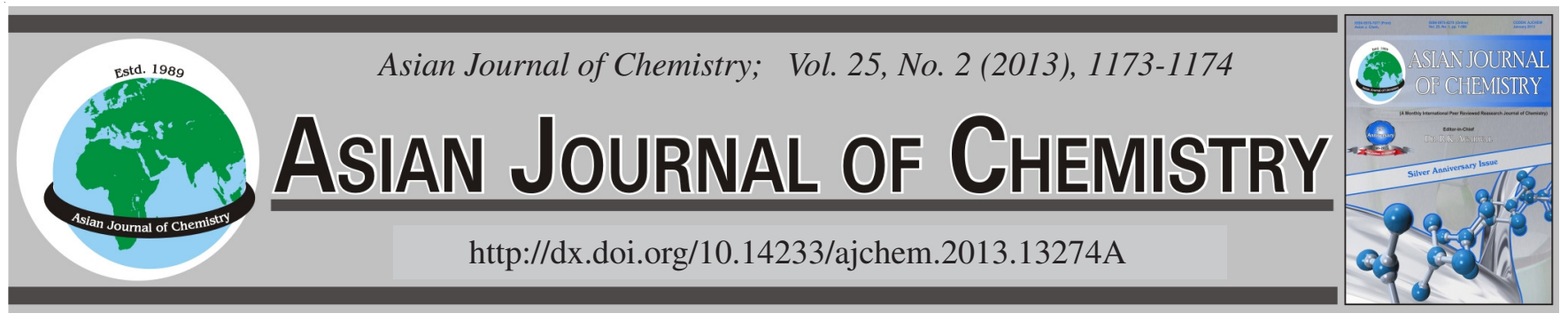

NOTE

\title{
Synthesis, Growth and Spectral Studies of Cadmium Mercury Thiocyanate Dimethyl Sulfoxide: An Efficient Non-linear Optical Single Crystal
}

\author{
Johanan Christian Prasana ${ }^{1}$, A. Anuradha ${ }^{2}$, J. Madhavan ${ }^{3, *}$ and P. Sagayaraj ${ }^{3}$
}

${ }^{1}$ Department of Physics, Madras Christian College, Chennai-600 059, India

${ }^{2}$ Department of Physics, Queen Mary's College, Chennai-600 004, India

${ }^{3}$ Department of Physics, Loyola College, Chennai-600 034, India

*Corresponding author: E-mail: jmadhavang@yahoo.com

(Received: 16 December 2011;

Accepted: 31 August 2012)

AJC-12073

The salt of tetrathiocyanato cadmium(II) mercury(II)-dimethyl sulfoxide was synthesized by using dimethyl sulfoxide as ligand to react
with cadmium mercury tetrathiocyanate (CMTC) in a mixture of dimethylsulfoxide and double distilled water. The grown crystals were
confirmed by single crystal XRD and it is found that cadmium(II) mercury(II)-dimethyl sulfoxide belongs to the orthorhombic system
with space group P $2_{1} 2_{1} 2_{1}$. The FTIR studies confirmed the coordination of dimethyl sulfoxide with Cd. The UV-Vis-NIR transmission
studies show the presence of a good transmission window which is the prime necessity for the material to have non-linear optical property. |
The non-linear optical efficiency of cadmium(II) mercury(II)-dimethyl sulfoxide crystal was studied and confirmed by Kurtz and Perry
technique and compared with other well-known non-linear optical crystals. It is interesting to observe that the non-linear optical effi-
ciency of cadmium(II) mercury(II)-dimethyl sulfoxide is superior to urea.
Key Words: Crystal growth, Second harmonic generation, Thermal studies.

The emergence of second order non-linear optical (SONLO) materials which are capable of efficient frequency conversion of infrared or visible laser radiation to visible or ultraviolet wavelengths have opened a new era in photonics technology. In the last decade, organic non-linear optical crystals with aromatic rings have gained more attention due to their high nonlinearity, fast response and tailor-made flexibility ${ }^{1}$.

However, organic non-linear optical crystals suffer due to the drawbacks such as, poor chemical stability, low hardness, red-shift of the cut-off wavelength caused by a large organic $\pi$-conjugated system. Hence in order to keep the merits and overcome the shortcomings of organic materials, a series of new organometallic or semiorganic complex non-linear optical crystals were developed ${ }^{2-4}$. These materials are found to possess good non-linear optical properties combined with moderate physico-chemical properties. In recent years, various scientific developments in the field of semiconductor materials have resulted in stable blue light emitting diodes (LED's). But stable output of violet light still could not be obtained. In order to obtain a stable output of violet light, which can be used to generate information storage, research on interesting category of organometallic and coordination complex non-linear optical materials have been attempted. Cadmium mercury thiocyanate dimethyl sulfoxide (CMTD) is one such potentially useful candidate of second order non-linear optical crystal with an empirical formula $\mathrm{C}_{8} \mathrm{H}_{12} \mathrm{~N}_{4} \mathrm{O}_{2} \mathrm{~S}_{6} \mathrm{CdHg}$. It is basically a Lewis base adduct of cadmium mercury thiocyanate dimethyl sulfoxide. The interesting feature in CMTD is attributed to the improvement of NLO property caused by the addition of DMSO ligand. The introduction of DMSO aids better electronic oscillations in SCN ligand, thus an improved NLO efficiency takes place. The crystal structure of CMTD is reported to be orthorhombic with space group $\mathrm{P} 2{ }_{1} 2_{1} 2_{1}$. In the present investigation, the growth of CMTD by slow solvent evaporation technique has been carried out and its physical properties are studied by various characterization techniques.

Synthesis and growth: Commercially available AR grade (E-Merck, purity $>98 \%$ ) chemicals were used as the starting materials. The synthesis of the title compound, cadmium mercury thiocyanate (CMTC) was carried out by carefully incorporating the stoichiometric amount of $\mathrm{NaSCN}, \mathrm{CdCl}_{2}$ and $\mathrm{HgCl}_{2}$ in the appropriate ratio using the chemical reaction given below:

$4 \mathrm{NaSCN}+\mathrm{CdCl}_{2}+\mathrm{HgCl}_{2} \longrightarrow \mathrm{CdHg}(\mathrm{SCN})_{4}+4 \mathrm{NaCl} \downarrow$

The resulting product appeared as a white precipitate, which was filtered and dried and then re-crystallized either in 
acetone solution or in water to improve the purity. During the second stage, the seed crystals were obtained by using dimethyl sulfoxide (DMSO) as a ligand to react with cadmium mercury thiocyanate (CMTC) in a mixture of dimethyl sulfoxide and de-ionized water. The following chemical reaction is expected to place.

$$
\mathrm{CdHg}(\mathrm{SCN})_{4}+2\left(\mathrm{CH}_{3}\right)_{2} \mathrm{SO} \longrightarrow \mathrm{CdHg}(\mathrm{SCN})_{4}\left(\mathrm{H}_{6} \mathrm{C}_{2} \mathrm{OS}\right)_{2}
$$

Within a period of 30-40 days, colourless, defect free and highly transparent good quality single crystal of dimension $11 \mathrm{~mm} \times 11 \mathrm{~mm} \times 8 \mathrm{~mm}$ was obtained by slow solvent evaporation method.

Single crystal X-ray diffraction analysis: It is observed from the X-ray diffraction data that the crystal system of $\mathrm{CdHg}(\mathrm{SCN})_{4}$ is orthorhombic with a non-centro symmetric space group $\mathrm{P} 2{ }_{1} 2_{1} 2_{1}$, with the lattice parameters $\mathrm{a}=8.5182 \AA$, $\mathrm{b}=8.5394 \AA, \mathrm{c}=28.227 \AA$ and cell volume $\mathrm{V}=2053.2 \AA^{3}$. The XRD data of present work are fairly in agreement with the reported work 5 .

FTIR studies: The FTIR spectrum of the grown crystal was recorded in the range of 4000-400 $\mathrm{cm}^{-1}$ using the instrument BRUKERS IFS-66V FTIR spectrometer. The highly intense and sharp peak observed at $2106 \mathrm{~cm}^{-1}$ corresponds to $\mathrm{CN}$ stretching. The absorption peak assigned to S-O stretching modes $\left(1008 \mathrm{~cm}^{-1}\right)$ appears at lower frequency and it compares well with those of DMSO due to the coordination of $\mathrm{O}$ atoms with the $\mathrm{Cd}$ atoms. The weak and sharp bands observed at 706 and $684 \mathrm{~cm}^{-1}$ are mainly attributed to CS stretching vibrations, which give rise to the probability of metal coordinations with SCN. Further, the bands at 2310 and $2562 \mathrm{~cm}^{-1}$ also witness chelating. Nevertheless, whether the chelation involves $\mathrm{Cd}$ or $\mathrm{Hg}$ ions and/or any metal-metal bonds are involved may not be answered categorically with the available data, as this information requires the recording of the far - IR spectrum of the sample to make it more authentic. It is interesting to see that the stretching and bending modes of $\mathrm{CH}_{3}$ are found shifted to 2915 and $1415 \mathrm{~cm}^{-1}$ from that of free DMSO molecules (2913 and $1406 \mathrm{~cm}^{-1}$ ). The above study confirms that the DMSO ligand is included in the crystal lattice of CMTC.

Optical transmission spectrum analysis: The optical transmission spectral analysis is an important study for any NLO material as it can be put into use only if it possesses the required cut-off wavelength as well as a wide optical transmission window. The UV-VIS-NIR spectrum of CMTD is shown in Fig. 1. It is observed from the figure that the sample has an optical transmission range from 380 to $2000 \mathrm{~nm}$ with almost uniform transmission in the entire region. Thus, the present study shows a wide transmission window for CMTD and thus indicating that the crystal may be used as a NLO material.

Thermal studies: The thermal analyses of CMTD single crystals were carried out using SDT Q600 thermal analyzer. From the DTG thermogram of CMTD, it is evident that the sample is stable up to $150.5^{\circ} \mathrm{C}$ and the sample successively undergoes three stages of decomposition. The first stage of decomposition reveals the decomposition of $\mathrm{CdHg}(\mathrm{SCN})_{4}(\mathrm{DMSO})_{2}$ into $\mathrm{CdHg}(\mathrm{SCN})_{4}$, in which two numbers of DMSO ligand molecules are lost. The second stage of decomposition is an

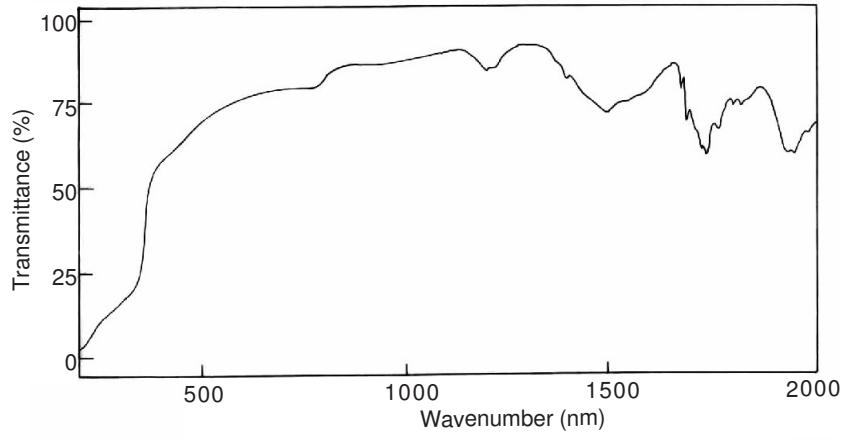

Fig. 1. Optical transmission spectrum of cadmium mercury thiocyanate dimethylsulfoxide

overlapping stage which extents from 250 to $950{ }^{\circ} \mathrm{C}$. In this stage, a high temperature weight loss occurs and this leads to the breakdown of three-dimensional steric structure and the release of volatile products of CMTC. In DTG trace, the peaks observed at $261.62,450.01$ and $738.86{ }^{\circ} \mathrm{C}$ might be attributed to the major decomposition of CMTD into the corresponding metal sulfides ( $\mathrm{CdS}$ and $\mathrm{HgS}$ ), carbon bisulfide $\left(\mathrm{CS}_{2}\right)$ and nitrogen molecules $\left(\mathrm{N}_{2}\right)$. The third stage of decomposition is observed between 750 and $900{ }^{\circ} \mathrm{C}$, which may be attributed to the oxidation process. Thus, it is observed from the thermal analysis that CMTD is thermally less stable when compared with CMTC6 $\left(198^{\circ} \mathrm{C}\right)^{6}$.

\section{Conclusion}

In the present study, organometallic complex NLO single crystals of CMTD are successfully grown in DMSO-water mixture solvent system by slow solvent evaporation technique. The grown crystals were confirmed by single crystal XRD. The FTIR study confirmed the coordination of DMSO with $\mathrm{Cd}$ through $\mathrm{O}$ atom and coordination of $\mathrm{Cd}$ and $\mathrm{Hg}$ metal ions with thiocyanate $(\mathrm{SCN})$ respectively. The microhardness study indicates that the CMTD belongs to the hard category of materials. The dielectric study revealed the low dielectric constant and dielectric loss of the crystal. The TG-DTG thermograms of CMTD confirm the starting of first stage decomposition at $154.5^{\circ} \mathrm{C}$, which is due to the loss of DMSO molecules and subsequently the sample decomposes as in the case of CMTC.

\section{REFERENCES}

1. J. Madhavan, S. Aruna, A. Anuradha, D. Premanand, I.V. Potheher, K. Thamizharasan and P. Sagayaraj, Opt. Mater, 29, 1211 (2007).

2 G.P. Joseph, K. Rajarajan, M. Vimalan, S. Selvakumar, S.M. Ravi Kumar, J. Madhavan and P. Sagayaraj, Mater. Res. Bull., 42, 2040 (2007).

3 K. Rajarajan, G. Mani, I.V. Potheher, J.G.M. Jesudurai, M. Vimalan, D. Christy, J. Madhavan and P. Sagayaraj, J. Phys. Chem. Solids, 68, 2370 (2007).

4 I.V. Potheher, J. Madhavan, K. Rajarajan, K.S. Nagaraj and P. Sagayaraj J. Cryst. Growth, 130, 124 (2008).

5 S. Guo, D. Xu, M.K. Lv, D.R. Yuan, Z.H. Yang, G.H. Zhang, S.Y. Sun, X.Q. Wang, M. Zhou and M.H. Jiang, Prog. Crystal Growth Charact., 40, 111 (2000).

6 X.Q. Wang, D. Xu, M.K. Lu, D.R. Yuan, J. Huang, S.G. Li, G.W. Lu, H.Q. Sun, S.Y. Guo, G.H. Zhang, X.L. Duan, H.Y. Liu and W.L. Liu, J. Cryst. Growth, 247, 432 (2003). 